\title{
Appliquer la télédetection lidar à la surveillance de l'air
}

L'éruption du volcan Eyjafjöll sur le sol islandais le 14 avril 2010 a perturbé pendant plusieurs semaines le trafic aérien, conduisant à l'annulation de près de 100000 vols et à une perte de près de 4 milliards d'euros par les compagnies aériennes et les professionnels du tourisme. Pour suivre la concentration en cendres dans I'air et prévenir les dommages aux avions en indiquant aux compagnies quand elle devenait

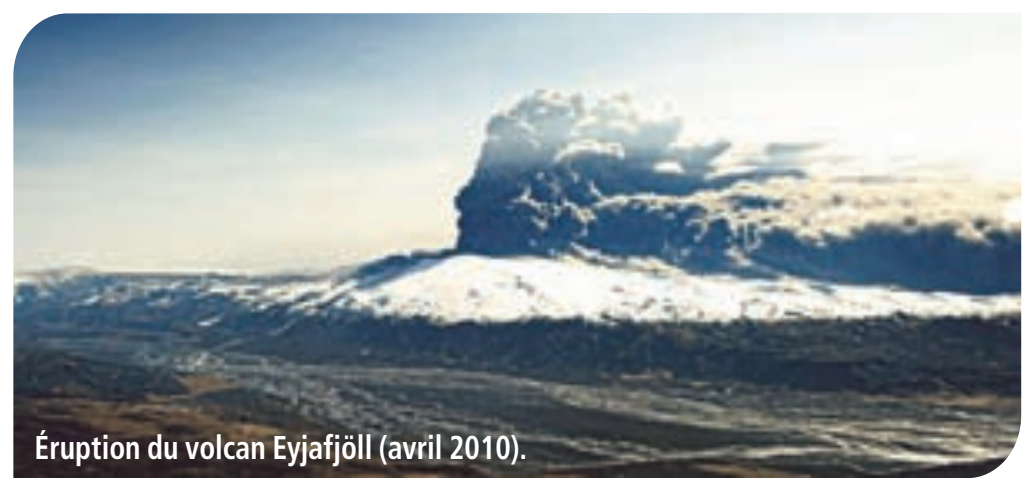
à nouveau assez basse pour des vols sans risque, un système lidar (Light Detection and Ranging) de nouvelle génération a été embarqué à bord d'un avion de recherche.

Patrick CHAZETTE

Laboratoire science du climat et de l'environnement (CEA)

patrick.chazette@lsce.ipsl.fr

\section{Des cendres qui voyagent...}

Lors de l'éruption du volcan, les cendres ont été injectées dans la colonne atmosphérique jusqu'à près de 10 kilomètres d'altitude au-dessus de I'Islande et se sont déplacées en altitude, sans pénétrer immédiatement dans les premières couches de l'atmosphère, celles où nous vivons, sauf aux abords du volcan comme on a pu le constater, par les grandes quantités de cendres recouvrant la pointe sud de l'Islande. En fonction des conditions météorologiques, les cendres peuvent se déplacer sur des milliers de kilomètres et la remontée de l'anticyclone des Açores a favorisé leur voyage vers l'Europe de l'Ouest.

C'est ainsi que le panache a atteint le nord de la France le 16 avril 2010 au cours de l'après midi, comme l'a observé le système lidar sol du site CEA de Saclay, au sud-ouest de Paris (figure 1) : les cendres sont arrivées en région parisienne à une

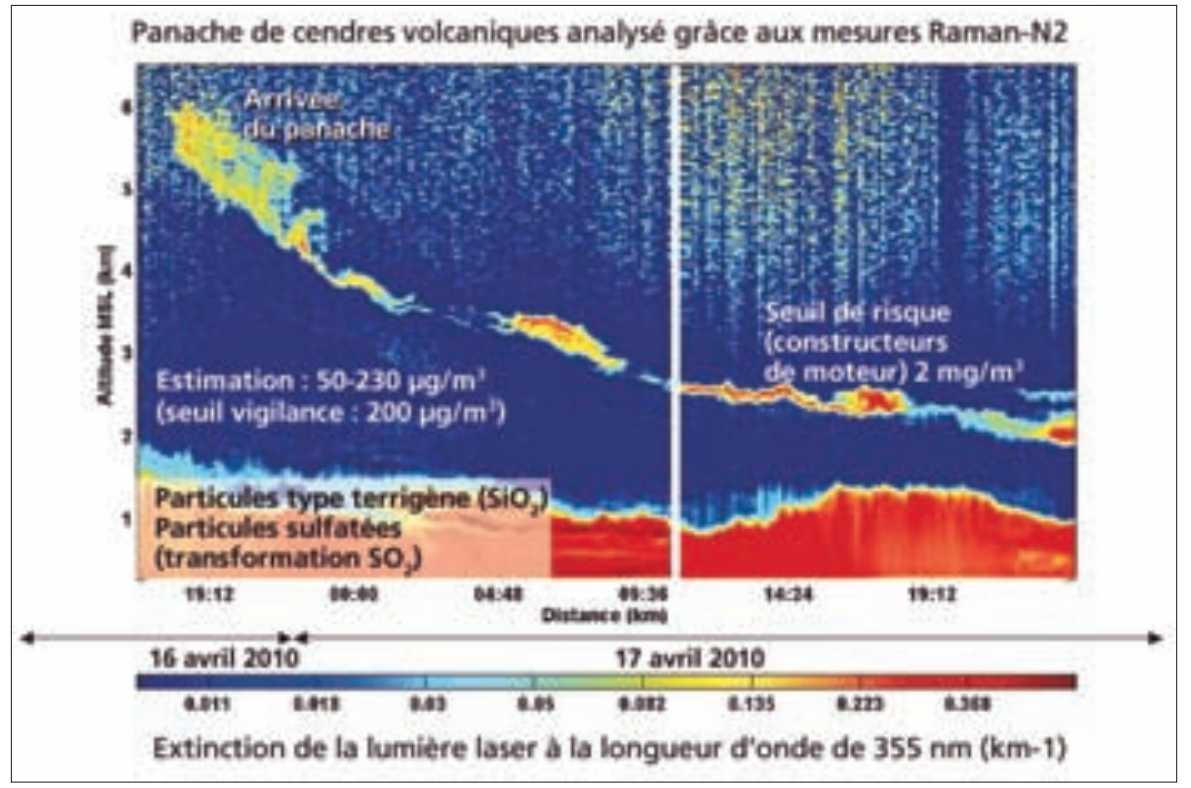

Figure 1. Propriétés d'extinction de la lumière laser à 355 nm dérivées du système lidar RamanN2 du site CEA de Saclay. Jusqu'à deux kilomètres d'altitude, la couche limite atmosphérique peut être observée (notez qu'elle est très polluée). Au-dessus, les cendres volcaniques apparaissent à des altitudes diminuant au cours du temps d'environ 6 à $2 \mathrm{~km}$. La concentration de cendres peut être évaluée en connaissant leur granulométrie et leur composition chimique. Les premières estimations donnent des valeurs entre 50 et $230 \mu \mathrm{g} / \mathrm{m}^{3}$ à l'arrivée du panache.

altitude proche de six kilomètres pour atteindre environ deux kilomètres les jours suivants.

\section{... et des avions cloués au sol}

Or, si l'air comporte trop de cendres volcaniques, ces dernières risquent d'encrasser significativement les réacteurs d'avion et donc les endommager : en fondant, la silice contenue dans ces particules composées de plus de $50 \%$ d'oxyde de silicium se dépose sur des parties sensibles des moteurs.

Lors des premières perturbations de l'espace aérien, entre les 16 et 21 avril, nous n'avions pas d'information sur les seuils de dommage, puis une valeur de 
$2 \mathrm{mg} / \mathrm{m}^{3}$ a été fournie par les motoristes et un seuil de vigilance à $200 \mu \mathrm{g} / \mathrm{m}^{3}$ a été adopté.

La question était de savoir comment estimer les concentrations de cendres dans le panache situé en altitude sans faire prendre de risque aux avions « renifleurs». La solution s'est portée sur des mesures de télédétection à distance : les observations lidar.

\section{La télédétection lidar}

L'invention des lidar date des années soixante, peu après celle des laser. En France, les premiers systèmes lidar ont été installés à l'observatoire de HauteProvence pour étudier la mésosphère et la stratosphère [2]. C'est en particulier grâce à ce type d'instrument que les concentrations d'ozone dans la stratosphère sont surveillées depuis la découverte du trou d'ozone en 1985.

En 2004, le CEA, avec la participation du CNRS, a mis au point un système lidar dédié à l'étude de la qualité de l'air et à la surveillance de l'atmosphère météorologique. Ce système est actuellement commercialisé par la société française Léosphère et vendu à l'échelle internationale. C'est un instrument en sécurité oculaire, facilement transportable en voiture, bateau ou avion... fonctionnant dans l'ultraviolet à la longueur d'onde de $355 \mathrm{~nm}$. Le lidar est composé d'un émetteur (un laser), d'un récepteur (un télescope ou une lunette) et d'une chaîne

\footnotetext{
Un volcan, deux volcans...

Le 8 juin 1783, le volcan Katla rentrait en éruption sur le sol islandais et injectait dans l'atmosphère une telle quantité de cendres que le climat d'une grande partie de l'Europe de l'Ouest en fut perturbé, conduisant à des hivers rigoureux et une baisse de la production agricole (on dit que cet évènement serait l'un des initiateurs de la révolution française [1]). C'est son voisin moins dévastateur, le Eyjafjöll, qui s'est réveillé le 14 avril.

Oui, moins dévastateur... mais annonciateur d'une nouvelle éruption du Katla ? Ses deux précédentes éruptions, en 1612 et 1821, avaient été suivies par l'éruption du Katla dans les deux années qui suivirent !
}

électronique d'acquisition (figure 2). Le laser est pulsé à une fréquence allant de quelques hertz à plusieurs milliers. L'émission laser est synchronisée avec la base de temps de l'électronique d'acquisition et le faisceau est émis vers
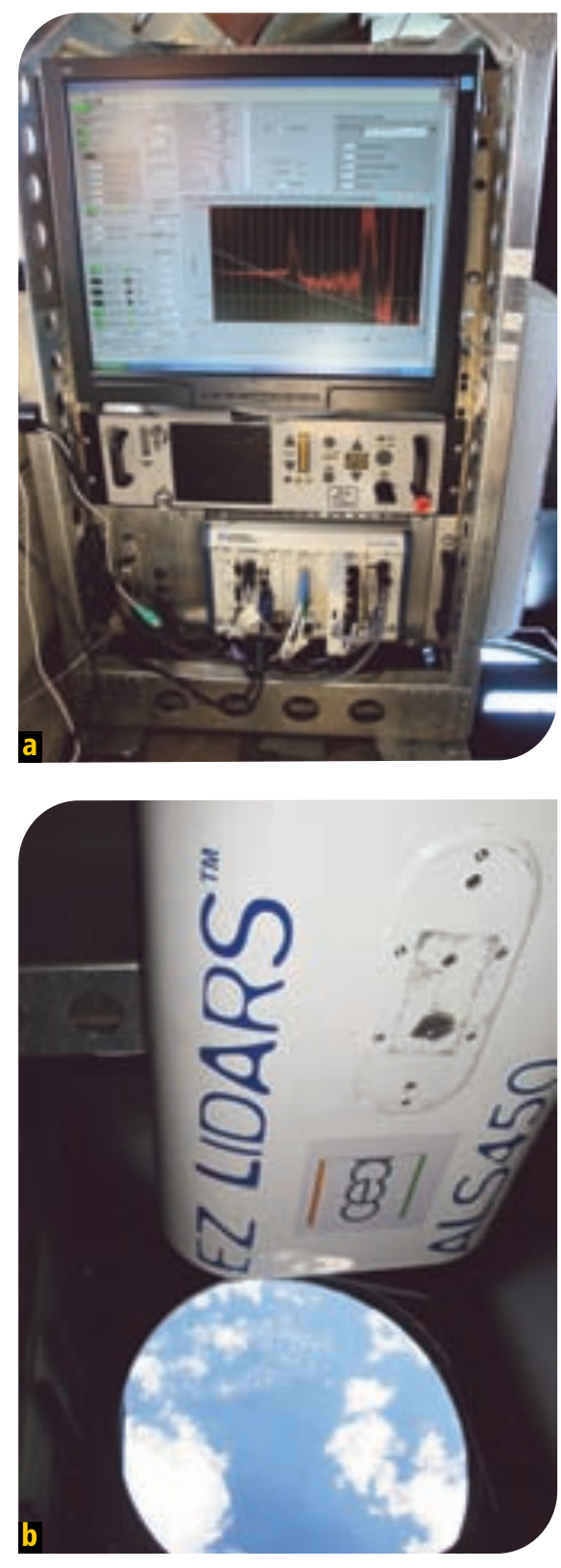

Figure 2. Vue du lidar installé dans le Falcon 20 de l'unité SAFIRE.

a. La chaîne d'acquisition avec un profil lidar (en rouge). Deux couches sont visibles et en fin de profil (le plus à droite), l'écho de sol sur I'Atlantique. Au centre de la baie, il y a l'électronique laser et en bas, le PC d'acquisition et de stockage des données lidar.

b. Le hublot en silice permet des tirs au nadir sans altérer la qualité du faisceau laser. La tête optique (émission et réception) est positionnée au dessus.

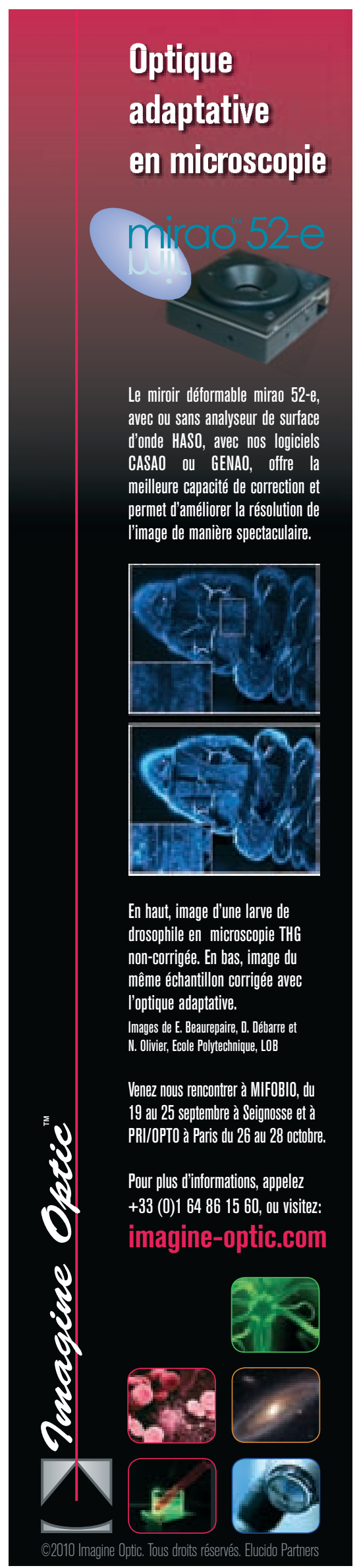


l'atmosphère où il interagit avec les molécules de l'air, les aérosols (les cendres) et les nuages. De cette interaction, une petite partie des photons incidents est rétrodiffusée vers le récepteur et convertie en tension par la chaîne d'acquisition. La résolution verticale du système lidar dépend de la largeur de la raie laser et de la fréquence d'échantillonnage choisie par l'utilisateur. Dans notre cas, la fréquence du laser est de $20 \mathrm{~Hz}$ et nous utilisons généralement des résolutions verticales comprises entre 1,5 et $30 \mathrm{~m}$.

\section{L'observation aéroportée du panache de cendres}

À la suite de l'arrivée du panache de cendres, à la demande des autorités gouvernementales, le CEA a adapté un système lidar pour l'embarquer sur l'avion Falcon 20 de I'unité SAFIRE (Service des avions français instrumentés pour la recherche en environnement) qui est une structure sous tutelle du CNRS, du CNES et de Météo-France.

Ces mesures lidar avaient pour objectif de localiser les couches résiduelles du panache de cendres volcaniques dans I'espace aérien français pour permettre aux compagnies aériennes de donner leurs autorisations de vol et valider la modélisation de la trajectoire du panache.

Le système lidar a été assemblé et configuré pour les vols avion le 19 avril. II a effectué son premier vol opérationnel le 21, à la fin de la première alerte. Le panache de cendres a néanmoins pu être très bien observé dans le nord de la France (figure 3). Pour le détecter, ce sont les propriétés de dépolarisation de la lumière laser par les cendres qui ont été utilisées : le laser émet dans une polarisation parallèle qui se trouve partiellement dépolarisée lors de son interaction avec les cendres. Ceci est dû au caractère non sphérique de ces particules. Dans la couche limite, comme dans les nuages, on observe également de la dépolarisation : elle est principalement due à la présence de particules

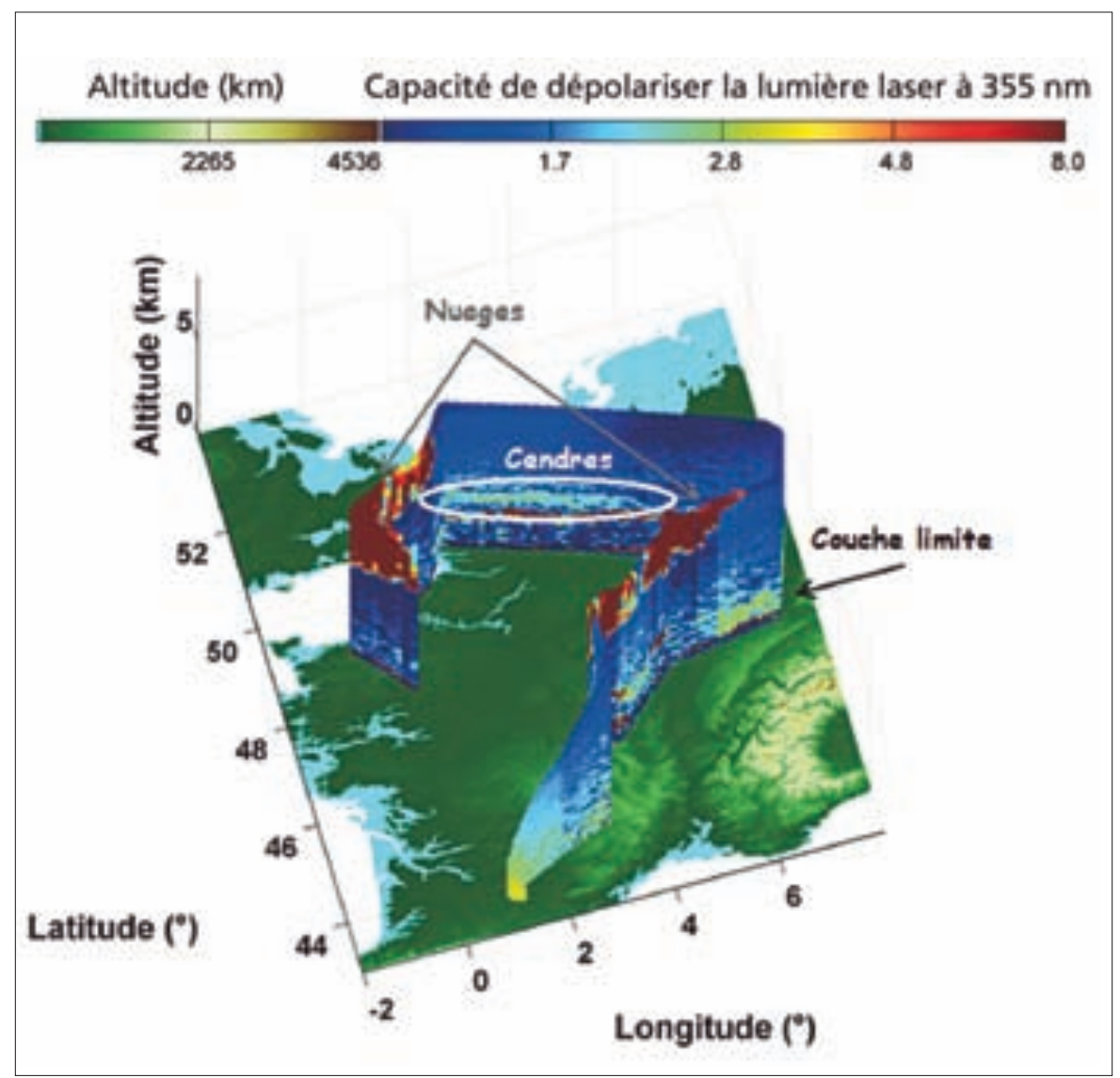

Figure 3. Observation du nuage de centre l'après midi du 21 avril 2010 à partir du lidar embarqué dans le Falcon 20. Les cendres sont très bien observées en mesurant la quantité de lumière laser dépolarisée.

terrigènes pour la couche limite (voir encadré) et à la diffusion multiple dans les structures nuageuses qui peuvent également contenir des cristaux de glace.

\section{Comment aller plus avant?}

Le lidar apporte des enseignements sur certaines propriétés optiques des cendres, mais ne permet pas à lui seul de déterminer leur nature chimique et leur concentration. Il faut pour cela disposer de mesures chimiques et granulométriques complémentaires réalisées, par exemple, par un autre avion qui volerait dans le panache. C'est ce qui a été fait avec I'ATR 42 de SAFIRE pour un panache de cendres peu dense. Les risques pour les moteurs d'avion étant important, il est également possible d'utiliser des mesures in situ lorsque les cendres s'approchent du sol.

La mise en place d'un réseau lidar opé- rationnel au sol est également d'actualité. Il permettrait de surveiller l'espace aérien national, même international, tout en apportant des contraintes fortes pour les prévisions d'évènements de pollution et l'étude des impacts climatiques liés à l'activité humaine.

Les particules terrigènes sont des particules de la surface de la Terre mises en suspension dans l'air suite à des processus mécaniques de soulèvement comme ceux liés à l'effet du vent (à l'instar des aérosols désertiques). Elles sont généralement de plus grande dimension (4 à $6 \mu \mathrm{m}$ de diamètre) que les aérosols de pollution (inférieur au micron). Elles sont non sphériques et dépolarisent la lumière laser.

\section{Références}

1. Emmanuel Garnier, Les dérangements du temps, 500 ans de chaud et de froid en Europe. Plon, 2010. 2. L'école de l'espace. Le service d'aéronomie 19582008. Editions du CNRS, 2008. 